\title{
Erratum to: Serum IL-33, a new marker predicting response to rituximab in rheumatoid arthritis
}

Jérémie Sellam ${ }^{1,10^{*}+}$, Elodie Rivière ${ }^{2 \dagger}$, Alice Courties ${ }^{1}$, Paul-Olivier Rouzaire $^{3}$, Barbara Tolusso ${ }^{4}$, Edward M. Vital ${ }^{5}$, Paul Emery ${ }^{5}$, Gianfranco Ferraccioli ${ }^{4}$, Martin Soubrier ${ }^{6}$, Bineta Ly ${ }^{2}$, Houria Hendel Chavez ${ }^{7}$, Yassine Taoufik Maxime Dougados ${ }^{8}$ and Xavier Mariette ${ }^{2, *^{*}}$

\section{Erratum}

Unfortunately, after publication of this article [1], it was noticed that the name of Gianfranco Ferraccioli was incorrectly spelled as Gianfranco Ferraciolli. The corrected author list can be seen above and the original article has been updated to correct this error.

\begin{abstract}
Author details
'Université Paris 06, AP-HP St-Antoine hospital, Rheumatology Department, INSERM UMRS_938, DHU i2B, Paris, France. ${ }^{2}$ Université Paris-Sud, AP-HP Hôpitaux Universitaires Paris-Sud, Rheumatology Department, Center for Immunology of Viral Infections and Autoimmune Diseases INSERM U1184, Le Kremlin Bicêtre, France. ${ }^{3}$ Biological Immunology Department, ERTICa Research Group, Clermont-Ferrand University Hospital, Clermont-Ferrand EA4677, France. ${ }^{4}$ Rheumatology Department, Catholic University of the Sacred Heart, Roma, Italy. ${ }^{5} \mathrm{NIHR}$ Leeds Musculoskeletal Biomedical Research Unit, Leeds Teaching Hospitals NHS Trust, Leeds, UK and Leeds Institute of Rheumatic and Musculoskeletal Medicine, University of Leeds, Leeds, UK. ${ }^{6}$ Rheumatology Department, Clermont-Ferrand University Hospital, Clermont-Ferrand, France. ${ }^{7}$ AP-HP Bicêtre Hospital, Biological Immunology Department, INSERM U1184, Le Kremlin Bicêtre, France. ${ }^{8}$ Department of Rheumatology - Hôpital Cochin, Paris Descartes University, Assistance Publique - Hôpitaux de Paris, INSERM (U1153), Clinical Epidemiology and Biostatistics, PRES Sorbonne Paris-Cité, Paris, France. ${ }^{9}$ Service de Rhumatologie, Hôpital de Bicêtre, 78 rue du Général Leclerc, Le Kremlin Bicêtre 94275, France. ${ }^{10}$ Service de Rhumatologie, Hôpital Saint-Antoine, 184 rue du Faubourg Saint-Antoine, Paris 75012, France.
\end{abstract}

Received: 6 January 2017 Accepted: 6 January 2017

Published online: 23 January 2017

\section{Reference}

1. Sellam J, Rivière E, Courties A, Rouzaire PO, Tolusso B, Vital EM, Emery P, Ferraccioli G, Soubrier M, Ly B, Hendel Chavez H, Taoufik Y, Dougados M, Mariette $X$. Serum IL-33, a new marker predicting response to rituximab in rheumatoid arthritis. Arthritis Res Ther. 2016;18:294. http://doi.org/10. 1186/s13075-016-1190-z.

\footnotetext{
* Correspondence: jeremie.sellam@aphp.fr; xavier.mariette@aphp.fr

${ }^{\dagger}$ Equal contributors

${ }^{1}$ Université Paris 06, AP-HP St-Antoine hospital, Rheumatology Department, INSERM UMRS_938, DHU i2B, Paris, France

2Université Paris-Sud, AP-HP Hôpitaux Universitaires Paris-Sud, Rheumatology Department, Center for Immunology of Viral Infections and Autoimmune Diseases INSERM U1184, Le Kremlin Bicêtre, France
} 